\title{
DL 假设下一种更高效的第三方权力受约束的 IBE 方案
}

\author{
徐鹏，崔国华*，付才，汤学明 \\ 华中科技大学计算机科学与技术学院信息安全实验室, 武汉 430074 \\ * 通信作者. E-mail: cgh3986@126.com
}

收稿日期: 2009-07-15; 接受日期: 2009-11-10

湖北省自然科学基金 (批准号: 2008CDB352) 和国家自然科学基金 (批准号: 60703048, 60903175) 资助项目

\begin{abstract}
摘要鉴于基于身份加密方案 (IBE 方案) 存在密钥托管问题, 2007 年 Goyal 创 造性的提出了一种新的方法, 即第三方权力受约束的基于身份加密方案 (A-IBE 方 案), 使得在一定程度上降低了用户对私钥生成方的信任需求. 在 Goyal 的基础上, 文中研究并提出了一种新的通用 A-IBE 方案. 通过分析新方案的安全性证明可以 看出: 和 Goyal 的方案相比, 新方案的安全性证明在一个更强的安全性定义(适应 性选择挑战身份信息)下, 基于一个更弱的难题 (离散对数问题) 实现了更 “紧”的 安全性规约. 因此, 相比 Goyal 的方案, 新方案可以选择一个更小的安全参数, 从 而在一定程度上提高计算效率. 在执行效率方面, 新方案尽可能的减少了“对”运 算的次数, 且该次数达到最优, 即不可能再减少该次数且不增加计算复杂性; 在总 的计算复杂度方面, 新方案在相当的程度上比 Goyal 的方案更优. 但遗憾的是, 这 些改进也会在一定程度上增加公开参数和通信量. 因此实际应用中，可以选取折 衰的或满足特定需求的公开参数的大小, 进而平衡利弊.
\end{abstract}

\section{1 引言}

基于身份加密 (identity-based encryption, IBE) 的概念是由 Shamir ${ }^{[1]}$ 于 1984 年首次提出的, 是 一种特殊的公钥密码体制. 和传统的公钥密码体制不同, 它通过采用能标识唯一身份的公开信息作为 公钥实现加密, 从而在很大程度上减少了对在线第三方的需要, 即解决了传统公钥密码体制中第三方 的性能瓶颈问题. 虽然 IBE 的概念很早即已提出, 但直到 2001 年, 第一个实用的 IBE 方案 ${ }^{[2]}$ 才被 Boneh 等人提出. 从此针对 IBE 方案的研究进入快速发展阶段. 2004 年, Boneh 等人在以前研究的基 础上, 提出了两个在标准模型下具有可证明安全性的 IBE 方案 ${ }^{[3,4]}$, 但是前者 ${ }^{[3]}$ 仅能在较弱的安全性 定义下具有有效的安全性归约, 而后者 ${ }^{[4]}$ 虽然可以在强安全性定义下具有有效的安全性归约, 但是却 在很大程度上增加了时间和空间复杂度. 目前为止, 综合考虑强安全性定义下可证明安全的归约性能 和时空复杂度, 最有效的两个 IBE 方案分别由 Waters ${ }^{[5]}$ 和 Gentry ${ }^{[6]}$ 于 2005 年和 2006 年提出, 但是 由于这两个方案分别基于不同的难题假设, 因此很难确定哪一个更优. 
对于 IBE 方案的研究, 除了提出更有效的方案外 (即更有效的安全性归约和时空复杂度), 为了实 现 IBE 方案最终的工业化, 依然存在许多问题需要解决, 其中最重要的问题之一是密钥托管问题 ${ }^{[2]}$ (目

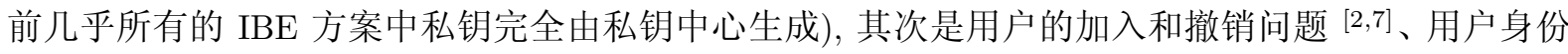
信息的提取和绑定问题 ${ }^{[8]}$ 等等.

IBE 方案的密钥托管问题是最重要的问题之一, 是决定着 IBE 方案实用性的重要因素. 由于 IBE 方案中用户的私钥一般完全由私钥中心 (private-key generator, PKG) 生成, 因此用户需要完全信任 PKG, 但是在实际应用中, 很难让用户做到这一点; 同时, PKG 本身也很难保证绝对的安全性. 因此面 对这些可能的极具破坏性的不安全因素, 即使 IBE 方案具有突出的方便快捷的性能优势, 实用中用户 恐怕也不会选择 IBE 方案来取代传统的公钥体制 (例如: 公钥基础设施 PKI). 因此如何解决 IBE 方 案的密钥托管问题成为热门研究之一. 目前有 3 种方案来解决该问题, 分别如下:

第 1 种方案: 用户的私钥通过限门技术由多个 PKG 共同生成 ${ }^{[2]}$. 这样即使少数的 PKG 被成功 攻破, 也不会泄露用户的私钥信息. 但是与此同时, 多 PKG 的存在使得系统结果更复杂、通信代价大 大增加.

第 2 种方案: 无证书公钥密码方案 (certificateless public-key encryption scheme, CL-PKE scheme) ${ }^{[9]}$. 该方案通过组合 IBE 方案和 PKI 体制的思想, 使得用户的私钥由两个部分共同组成, 且这两个部分 分别独立的由 PKG 和用户生成, 这样 PKG 并不知道整个私钥的内容. 并且和 PKI 体制不同的是, CL-PKE 方案的 PKG 不在发布与用户所选取的部分私钥相对应的部分公钥的证书, 而是直接发布该 部分公钥, 即减少了证书生成过程. 虽然 CL-PKE 方案从根本上解决了密钥托管问题, 但是它却增加 了对在线第三方的要求, 来响应发送方对接收方部分公钥的询问. 虽然 CL-PKE 是一个不错的解决方 案, 但是显然它在很大程度上违背了 IBE 方案的意义.

2007 年, Goyal 创造性的提出了第三种解决方案 [10]. 该方案在不改变 IBE 方案的基础结构的 条件下, 减少了用户对 PKG 的信任需求, 且该方案称为第三方权利受约束的 IBE 方案 (accountable authority IBE scheme, A-IBE scheme). 总的来说, 在 A-IBE 方案的密钥生成协议中, 当用户收到来自 PKG 的私钥种子信息的时候, 用户通过秘密选取 “迹” 信息来部分的决定用户私钥的生成. 因此, 从 直觉上来说, 由于 PKG 并不知道用户秘密选取的 “迹”, 因此也就无法独立生成具有相同 “迹”的该 用户的私钥. 另一方面, 由于私钥种子信息的秘密性, 用户在没有 PKG 的帮助下也无法额外的生成不 同 “迹” 的私钥. 因此在实际应用中, 若某用户发现有人知道他的一个有效的且不同 “迹” 的私钥时, 那么该用户就可以充分的认定 PKG 伪造了他的私钥, 从而进行高额索赔. 较具体地来说, 文献 [10] 首次给出了 A-IBE 的定义及其特殊的安全性定义, 并且分别基于 Gentry ${ }^{[6]}$ 和 Waters ${ }^{[5]}$ 的 IBE 方案, 构造了两个具体的可证明安全的 A-IBE 方案, 而且为了更有效的发现实际应用中的欺骗行为, 提出了 可以发现解密服务提供者的黑盒型 A-IBE 方案 (black-box accountable authority IBE scheme, BA-IBE scheme), 但是当前该类方案还只能在较弱的模型 [10] 下构建有效的 BA-IBE 方案, 因此如何在完整的 模型下构建该方案成为公开的问题.

下面, 在分析文献 [10] 中 Goyal 提出的第二个 A-IBE 方案的基础上, 将列举出本文的贡献.

文献 [10] 中, Goyal 提出的第二个 A-IBE 方案是一个通用方案 (该方案以后简称为 A-IBE-G2 方 案), 即任何安全的 IBE 方案都可以被扩展为一个安全的 A-IBE 方案, 额外地也可以在较弱的模型下 扩展为 BA-IBE 方案.

与 A-IBE-G2 方案相比, 本文的贡献如下:

1) 通过尽可能地减少对运算 ${ }^{[2,11]}$ 的次数, 本文将提出一个新的通用 A-IBE 方案, 且新方案中对运 
算只在密文有效性验证阶段发生. 更进一步地说, 就我们所知, 该阶段的 “对” 运算具有必要性,即不可 能再减少该处的对运算而不增加计算复杂性. 因为对运算是目前最有效的可以解判定的 Diffie-Hellman (decisional Diffie-Hellman, DDH) 问题,而不破坏计算的 Diffie-Hellman (computational Diffie-Hellman, $\mathrm{CDH})$ 问题 ${ }^{[12]}$ 的难解性的技术. 而众所周知的其他具有该特性的技术, 如: 零知识证明等通常都是非 常复杂和低效的. 另外, 本文相应章节还将解释为什么对运算的次数已经最优.

2) 对比新方案和 A-IBE-G2 方案在安全性定义 ComputeNewKey game ${ }^{[10]}$ 下的安全性归约, 可以 看出: 本文提出的新方案在更强的安全性定义 (在攻击者完成私钥询问后, 攻击者可以适应性选择挑 战 ID) 下, 基于一个更弱的难题 (离散对数问题 ${ }^{[13]}$ ) 实现了更有效的归约; 相反地, A-IBE-G2 仅实现 了较弱安全性定义下的安全性归约, 即攻击者在进行私钥询问前, 需要选择挑战 ID; 在归约程度方面, 对于每一次的私钥询问, A-IBE-G2 方案的安全性归约中仿真器的失败概率为 $1 / 2$, 因此造成了较大的 安全性退化 ${ }^{[14]}$, 而为了弥补这一点实用中需要选取较大的安全参数; 而同样是归约程度方面, 本文提 出的新方案使得其安全性归约中仿真器的失败概率为 $1 / n$, 其中 $n$ 由用户选取, 且当 $n$ 等于攻击者进 行私钥询问的次数时该归约效率最优, 但是由于本文提出的新方案的公开参数的大小与 $n$ 成正比, 因 此过大的 $n$ 也将造成公开参数存储量的增加. 也正是由于这一点, 实用中如何选取适当大小的 $n$ 也和 应用的实际需求相关.

本文组织结构如下: 第 2 节将介绍和提出一些有关的必要知识; 第 3 节将给出新的通用 A-IBE 方 案; 第 4 节将给出新的通用 A-IBE 方案的安全性证明; 第 5 节将从多个方面对比新的通用 A-IBE 方 案和已有的 A-IBE-G2 方案; 最后, 第 6 节对全文进行总结 (除特殊说明外, 本文中所有的符号和简称 与第一次出现时的含义相同).

\section{2 预备知识}

\section{1 难题假设}

离散对数假设 (discrete logarithm assumption, DL assumption) ${ }^{[13]}$ 是一个经典的难题, 很多广泛使 用的难题都可以高效的归约到该难题, 例如 $\mathrm{CDH}$ 假设、DDH 假设 ${ }^{[12]}$ 等. 因此抽象地说, 与基于其他 较强的难题且具有可证明安全性的方案相比,一个基于 DL 假设的可证明安全的方案通常更加安全.

定义 1 离散对数假设. 令 $G_{1}$ 为大素数 $p$ 阶群, 且 $g$ 是它的生成元, 随机选取 $a \in \mathbb{Z}_{p}$. 离散对 数问题是指: 输入 $\left\langle g, g^{a}\right\rangle$, 求解 $a$. 离散对数假设是指: 不存在概率多项式时间算法可以以不可忽略的 概率求解离散对数问题.

\section{2 有关通用 A-IBE 方案的若干定义}

\subsection{1 通用 A-IBE 方案}

根据 Goyal 的研究 ${ }^{[10]}$, 本文将 A-IBE 方案分成两类: 一类是特殊的 A-IBE 方案; 一类是通用 A-IBE 方案. 文献 [10] 提出的第一个 A-IBE 方案即为特殊的 A-IBE 方案, 它主要通过修改满足特定 条件的 IBE 方案的 Extract 算法实现 A-IBE 的功能和安全性需求, 但是仅有少数 IBE 方案可以 (据 我们所知, 目前仅有 Gentry 提出的 IBE 方案 [6] 可行). 文献 [10] 提出的第二个 A-IBE 方案即为通用 A-IBE 方案, 它可以将任何 IBE 方案通过并行的链接一个新的部分将其扩展为 A-IBE 方案.

在文献 [10] 的基础上, 结合 Shamir 对 IBE 方案的定义 ${ }^{[2]}$, 本文将首次给出通用 A-IBE 方案的定 
义. 令 IBE 方案由 4 个算法组成, 且分别是: 算法 Setup、Extract、Encrypt 和 Decrypt, 则通用 A-IBE 方案由 5 个算法组成, 且分别定义如下:

Setup $-u A$ 算法: 以安全参数 $k$ 作为唯一的输入; 对任意取定的 IBE 方案 $I$, 随机的生成公开参 数 $\mathrm{PK}=\{\mathrm{PK}-I, \mathrm{PK}-A\}$ 和主密钥 $\mathrm{MK}=\{\mathrm{MK}-I, \mathrm{MK}-A\}$, 其中 $\mathrm{PK}-I$ 和 $\mathrm{MK}-I$ 分别为 $I$ 的 公开参数和主密钥, 且由 $I$ 的 $\operatorname{Setup}(k)$ 算法生成.

KeyGeneration 协议: 取身份信息 ID 和公开参数 PK 为公共输入, 主密钥 MK 为 PKG 的私有输 入; PKG 和用户间通过运行该协议, 最终用户得到其私钥 $d_{\mathrm{ID}}=\left\{d_{I}, d_{A}\right\}$, 其中 $d_{I}$ 由 PKG 运行 $I$ 的 Extract(ID, PK $-I, \mathrm{MK}-I)$ 算法生成.

Encrypt $-u A$ 算法: 取身份信息 ID、公开参数 $\mathrm{PK}$ 和明文 $m$ 为输入; 在明文空间中随机选 取 $m_{1}$, 使得 $m=m_{1} \oplus m_{2}$, 其中 $m_{2}$ 也属于明文空间; 该概率算法输出密文 $C=\left\{C_{1}, C_{2}\right\}$, 其中 $C_{1}=\operatorname{Encrypt}\left(\mathrm{ID}, \mathrm{PK}-I, m_{1}\right), C_{2}$ 是 $m_{2}$ 的密文.

Decrypt $-u A$ 算法: 该确定算法以用户私钥 $d_{\mathrm{ID}}$ 、公开参数 $\mathrm{PK}$ 和密文 $C=\left\{C_{1}, C_{2}\right\}$ 作为输入; 输出明文 $m=m_{1} \oplus m_{2}$, 其中 $m_{1}=\operatorname{Decrypt}\left(d_{I}, \mathrm{PK}-I, C_{1}\right), m_{2}$ 是 $C_{2}$ 的明文.

Trace 算法: 取用户私钥作为输入, 该确定算法输出该私钥的特征信息.

注意, 在有些情况下, 若明文空间为 Setup $-u A$ 算法所生成的某个代数系统, 则上述定义中所有 的异或操作要修改为该代数系统中的操作, 例如: 若某 Setup $-u A$ 算法实例生成的代数系统为大素数 阶乘法群, 则上述定义中所有的异或操作要修改为该群的乘法运算.

\subsection{2 安全性定义}

从通用 A-IBE 的定义可以看出, 由于通用 A-IBE 方案并没有改变其选取的 IBE 方案, 而只是并 行的在所选 IBE 方案的基础上增加了一个新的部分, 因此通用 A-IBE 方案的语义安全性完全由选取 的 IBE 方案保证, 即若所选取的 IBE 方案是语义安全的, 则通用 A-IBE 方案同样具有该语义安全性. 因此本文并不考虑通用 A-IBE 方案的语义安全性.

通用 A-IBE 方案的核心安全性由安全性定义: FindKey game 和 ComputeNewKey game 组成 (具 体的定义参考文献 [10]). 安全性定义 FindKey game 下的有效安全性证明表示: 对于给定的挑战 ID, 在没有用户的帮助下, 敌对的 PKG 无法以不可忽略的概率生成一个新的该用户的私钥, 且该新生成 的私钥和原有私钥具有相同的特征信息. 另一方面, 安全性定义 ComputeNewKey game 下的有效安全 性证明表示: 敌对的用户即使可以询问除挑战 ID 以外的任何用户的私钥, 他也无法以不可忽略的概 率生成挑战 ID 的新的私钥, 且该私钥和原有私钥具有不同的特征信息, 换句话说敌对的用户无法诬 陷诚实的 PKG.

因此, 本文将仅考虑上述两种核心的安全性.

\section{$2.31 / n$ 不经意传输}

与 $1 / 2$ 不经意传输 $\left(O T_{2}^{1}\right)$ 类似, $1 / n$ 不经意传输 $\left(O T_{n}^{1}\right)^{[15,16]}$ 允许接收者从发送者所给的 $n$ 个秘 密中选取其中一个, 且发送者无法知道接收者选择了哪一个, 同时接收者也无法知道其他的秘密. $O T_{n}^{1}$ 可以由 $O T_{2}^{1}$ 通过通用的方法构成 ${ }^{[16]}$, 也可以通过密码技术直接构造 ${ }^{[15]}$. 有关 $O T_{n}^{1}$ 更多的内容请参 考上述文献. 


\section{3 新的通用 A-IBE 方案}

对于任意取定的且具有语义安全性的 IBE 方案 $I$, 新的通用 A-IBE 方案构造如下:

令 $G_{1}$ 是具有对运算的大素数 $p$ 阶群, $g$ 为其生成元; 令群 $G_{1}$ 上的对运算为 $e: G_{1} \times G_{1} \rightarrow G$; 令 $\sum=\{1, \ldots, n\}$ 是大小为 $n$ 的字母表; 令身份信息 $\mathrm{ID} \in \Sigma^{l_{\mathrm{ID}}}, l_{\mathrm{ID}}$ 为身份信息的长度, $\mathrm{ID}_{i}$ 表示 ID 的 第 $i$ 个字母; 令 $l_{s}$ 是关于安全参数 $k$ 的多项式. 令 $l=l_{\mathrm{ID}}+l_{s}, S_{\mathrm{ID}}=\left\{1, \ldots, l_{I D}\right\}, S_{s}=\left\{l_{\mathrm{ID}}+1, \ldots, l\right\}$.

Setup $-u A$ 算法: 取唯一的输入: 安全参数 $k$; 运行 $I$ 的 $\operatorname{Setup}(k)$ 算法, 得到 $I$ 的公开参数 PK $-I$ 和主密钥 $\mathrm{MK}-I ;$ 随机选取 $y \in \mathbb{Z}_{p}$ 和 $n \times l$ 阶主矩阵

$$
\boldsymbol{M} \boldsymbol{M}=\left(\begin{array}{ccc}
t_{1,1} & \ldots & t_{1, l} \\
\vdots & & \vdots \\
t_{n, 1} & \cdots & t_{n, l}
\end{array}\right)
$$

其中所有的 $t_{i, j} \in \mathbb{Z}_{p}$ 均不相同; 相应地, 计算公开矩阵

$$
\boldsymbol{P M}=\left(\begin{array}{ccc}
T_{1,1}=g^{t_{1,1}} & \ldots & T_{1, l}=g^{t_{1, l}} \\
\vdots & & \vdots \\
T_{n, 1}=g^{t_{n, 1}} & \ldots & T_{n, l}=g^{t_{n, l}}
\end{array}\right)
$$

发布公开参数 $\mathrm{PK}=\{\mathrm{PK}-I, \mathrm{PK}-A\}$, 其中 $\mathrm{PK}-A=\left\{\boldsymbol{P} \boldsymbol{M}, g^{y}\right\}$, 保密主密钥 $\mathrm{MK}=\{\mathrm{MK}-I, \mathrm{MK}-A\}$, 其中 $\mathrm{MK}-A=\{\boldsymbol{M} \boldsymbol{M}, y\}$. 注意: 该方案的明文空间可以合理的认为是群 $G_{1}$.

KeyGeneration 协议: 该协议由 PKG 和用户 $U($ 身份信息为 ID) 按如下过程实施:

1) PKG 随机地选取 $l$ 个数: $\left\{r_{1}, \ldots, r_{l}\right\} \in\left(\mathbb{Z}_{p}\right)^{l}$, 且使得 $r_{1}+\cdots+r_{l}=y$ 成立.

2) 对任意的 $i \in S_{\mathrm{ID}}$ : PKG 从主矩阵 $\boldsymbol{M} \boldsymbol{M}$ 中选取元素 $t_{\mathrm{ID}_{i}, i}$, 计算并返回 $r_{i} / t_{\mathrm{ID}_{i}, i}$ 给 $U$.

3) 对任意的 $i \in S_{s}$ : PKG 计算 $n$ 个元素: $\left\{r_{i} / t_{1, i}, \ldots, r_{i} / t_{n, i}\right\}$, 并作为其运行 $O T_{n}^{1}$ 协议的私有输 入, 其中 $\left\{t_{1, i}, \ldots, t_{n, i}\right\}$ 来自于主矩阵 $M \boldsymbol{M} ; U$ 秘密的选取 $d_{i} \in \Sigma$, 并将其作为其运行 $O T_{n}^{1}$ 协议的私 有输入; PKG 和 $U$ 运行 $O T_{n}^{1}$ 协议, 使得 $U$ 仅得到秘密 $r_{i} / t_{d_{i}, i}$, 且 PKG 无法知道 $d_{i}$.

4) $U$ 计算其私钥 $d_{A}=\left\{\left\{r_{i} / t_{\mathrm{ID}_{i}, i}\right\}_{i \in S_{I D}},\left\{d_{i}, r_{i} / t_{d_{i}, i}\right\}_{i \in S_{s}}\right\}$, 并且进行密钥有效性检测, 即检测

$$
g^{y}=\prod_{i \in S_{\mathrm{ID}}}\left(T_{\mathrm{ID}_{i}, i}\right)^{r_{i} / t_{\mathrm{ID}_{i}, i}} \prod_{i \in S_{s}}\left(T_{d_{i}, i}\right)^{r_{i} / t_{d_{i}, i}}
$$

若等式不成立, 则 $U$ 退出.

5) 对于 $U$ 的身份信息 ID, PKG 根据 $I$ 的参数 $\{\mathrm{PK}-I, \mathrm{MK}-I\}$, 运行 $I$ 的 Extract 算法计算其 部分私钥 $d_{I}$ 并返回给 $U$. 最后 $U$ 将 $d_{\mathrm{ID}}=\left\{d_{I}, d_{A}\right\}$ 作为其完整的私钥.

Encrypt $-u A$ 算法: 取身份信息 ID、公开参数 PK 和明文 $m \in G_{1}$ 作为输入, 该随机化算法按如 下过程计算密文 $C=\left\{C_{1}, C_{2}\right\}:$

1) 随机选取 $m_{1} \in G_{1}$, 令 $m=m_{1} \cdot m_{2}$, 其中 $m_{2} \in G_{1}$.

2) 运行 $I$ 的 Encrypt 算法, 计算 $\left.C_{1}=\operatorname{Encrypt(ID,PK}-I, m_{1}\right)$.

3) $C_{2}$ 是 $m_{2}$ 的密文且 $C_{2}=\left(C^{\prime \prime}=g^{s}, C^{\prime}=g^{s y} \cdot m_{2},\left\{C_{i}^{\prime}=\left(T_{\mathrm{ID}_{i}, i}\right)^{s}\right\}_{i \in S_{\mathrm{ID}}},\left\{C_{i, j}=\left(T_{i, j}\right)^{s}\right\}_{i \in \Sigma, j \in S_{s}}\right)$, 其中 $s$ 在 $\mathbb{Z}_{p}$ 中随机选取. 
Decrypt $-u A$ 算法: 该确定算法以私钥 $d_{\mathrm{ID}}$ 、公开参数 $\mathrm{PK}$ 和密文 $C=\left\{C_{1}, C_{2}\right\}$ 作为输入, 分别 通过解密 $C_{1}$ 和 $C_{2}$, 计算出 $m_{1}$ 和 $m_{2}$, 其中 $m_{1}=\operatorname{Decrypt}\left(d_{I}, \mathrm{PK}-I, C_{1}\right)$ (Decrypt 算法是 $I$ 的解密 算法), $C_{2}$ 的解密过程如下:

1) 首先运行密文有效性检测, 即检测 $\forall i \in S_{\mathrm{ID}}, e\left(C_{i}^{\prime}, g\right)=e\left(C^{\prime \prime}, T_{\mathrm{ID}_{i}, i}\right) ; \forall i \in \Sigma$ 和 $j \in S_{s}, e\left(C_{i, j}^{\prime}, g\right)$ $=e\left(C^{\prime \prime}, T_{i, j}\right)$; 若有任意的一个等式不成立, 则输出 $\perp$ (该符号在文中表示算法出现异常并停机). 由于 通用 A-IBE 方案的语义安全性完全继承于其选取的 IBE 方案的语义安全性, 因此此处的密文有效性 检测并不关心整个的密文 $C_{2}$, 而只关心其最后的两个部分, 即 $\left(\left\{C_{i}^{\prime}\right\}_{i \in S_{\mathrm{ID}}},\left\{C_{i, j}\right\}_{i \in \Sigma, j \in S_{s}}\right.$ ) 的有效性 (显 然, 在 $\mathrm{CCA}$ 安全性下, 此做法无法保证语义安全性, 因此进一步地说明 $C_{2}$ 并不需要在意语义安全性 问题). 而此处的密文有效性检测主要是为了保证：即使攻击者可以适应性的选择密文进行解密询问, 他也无法知道 $d_{\mathrm{ID}}$ 中有关 $d_{i}$ 的信息. 另一方面, 和 A-IBE-G2 方案一样, 该密文有效性检测中的对运 算可以减少为两次 (具体的细节参考文献 $[10]$ 的附录 $\mathrm{A}$ ).

2) 计算 $m_{2}$ 的方式如下:

$$
\frac{C^{\prime}}{\prod_{i \in S_{\mathrm{ID}}}\left(C_{i}^{\prime}\right)^{r_{i}} / t_{\mathrm{ID}_{i}, i} \prod_{i \in S_{s}}\left(C_{d_{i}, i}^{\prime}\right)^{r_{i}} / t_{d_{i}, i}}=\frac{g^{s y} \cdot m_{2}}{\prod_{i \in S_{\mathrm{ID}}} g^{s r_{i}} \prod_{i \in S_{s}} g^{s r_{i}}}=m_{2} ;
$$

最后输出 $m=m_{1} \cdot m_{2}$.

Trace 算法: 取私钥 $d_{\mathrm{ID}}$ 作为输入, 该确定算法输出 $\operatorname{Trace}\left(d_{\mathrm{ID}}\right)=d_{l_{\mathrm{ID}}+1}\|\cdots\| d_{l}$, 即私钥的特征值 为所有 $d_{i}$ 的串联.

由于很容易验证该新的通用 A-IBE 方案的正确性, 因此此处省略该过程.

\section{4 安全性证明}

本节将给出安全性定义 FindKey game 和 ComputeNewKey game 下, 新的通用 A-IBE 方案的安 全性证明.

定理 1 假设 $O T_{n}^{1}$ 协议是安全的, 则在安全性定义 FindKey game 下, 攻击者成功攻破新的通用 A-IBE 方案的优势可忽略.

证明 显然可以看出, 若攻击者可以生成新的私钥, 且和原有私钥 $d_{\mathrm{ID}}$ 具有相同的特征信息, 则 该攻击者已经成功的猜测了 $d_{l_{\mathrm{ID}}+1}\|\cdots\| d_{l}$. 而且由于在新的通用 A-IBE 方案的 KeyGeneration 协议 中, 所有 $d_{l_{\mathrm{ID}}+1}\|\cdots\| d_{l}$ 的 $d_{i}$ 均由用户 $U$ 秘密选取, 且仅在 $l_{s}$ 次 $O T_{n}^{1}$ 协议的运行中作为 $U$ 的秘密输 入使用, 因此该攻击者在成功猜测了 $d_{l_{\mathrm{ID}}+1}\|\cdots\| d_{l}$ 的同时, 也就破解了 $O T_{n}^{1}$ 协议的安全性. 但是这 和 $O T_{n}^{1}$ 协议是安全的假设相矛盾, 因此攻击者无法成功的猜测 $d_{l_{\mathrm{ID}}+1}\|\cdots\| d_{l}$, 也就无法破坏新的通 用 A-IBE 方案的 FindKey game 安全性, 即在安全性定义 FindKey game 下, 攻击者成功攻破新的通 用 A-IBE 方案的优势可忽略.

定理 2 假设 DL 假设成立, 则在安全性定义 ComputeNewKey game 下, 攻击者成功攻破新的通 用 A-IBE 方案的优势可忽略.

证明 假设存在攻击者 $A$ 可以以不可忽略的概率有效的生成挑战 ID 的两个有效的私钥 $d_{\mathrm{ID}}^{1}$ 和 $d_{\mathrm{ID}}^{2}$, 且 $\operatorname{Trace}\left(d_{\mathrm{ID}}^{1}\right) \neq \operatorname{Trace}\left(d_{\mathrm{ID}}^{2}\right)$, 那么可以构造一个有效的算法 $B$ 以不可忽略的概率求解 $\mathrm{DL}$ 问题.

令群 $G_{1}$ 上的 DL 问题为: 给定 $g^{a}$, 其中 $a$ 在 $\mathbb{Z}_{p}$ 中随机选取, 计算 $a$. 则算法 $B$ 按攻击者 $A$ 的 不可忽略优势如下求解 DL 问题: 
Setup 阶段: 算法 $B$ 运行新的通用 A-IBE 方案的 Setup $-u A$ 算法, 生成公开参数 $\mathrm{PK}=\{\mathrm{PK}-$ $I, \mathrm{PK}-A\}$ 和主密钥 $\mathrm{MK}=\{\mathrm{MK}-I, \mathrm{MK}-A\}$, 其中 $\mathrm{MK}-A=\{\boldsymbol{M} \boldsymbol{M}, y\}$,

$$
\boldsymbol{M M}=\left(\begin{array}{cccc}
t_{1,1} & \cdots & t_{1, l-1} & t_{1, l} \\
\vdots & & \vdots & \vdots \\
t_{n-1,1} & \cdots & t_{n-1, l-1} & t_{n-1, l} \\
t_{n, 1} & \cdots & t_{n, l-1} & \text { Null }
\end{array}\right)
$$

$\mathrm{PK}-A=\left\{\boldsymbol{P} \boldsymbol{M}, g^{y}\right\}$ 和

$$
\boldsymbol{P M}=\left(\begin{array}{ccc}
T_{1,1}=g^{t_{1,1}} & \ldots & T_{1, l}=g^{t_{1, l}} \\
\vdots & & \vdots \\
T_{n, 1}=g^{t_{n, 1}} & \ldots & T_{n, l}=g^{a}
\end{array}\right)
$$

注意: 和真实的新的通用 A-IBE 方案相比, 该阶段唯一的不同是主矩阵 $M M$ 的第 $n$ 行、 $l$ 列元 素. 且除此之外, 所有的参数和真实的新的通用 A-IBE 方案具有相同的分布, 即不可区分. 最后算法 $B$ 公布 $\mathrm{PK}=\{\mathrm{PK}-I, \mathrm{PK}-A\}$ 给 $A$.

KeyGeneration 阶段：该阶段攻击者可以通过与算法 $B$ 运行 KeyGeneration 协议, 询问到任意用 户的私钥. 对于攻击者 $A$ 询问的任意身份信息 ID, 算法 $B$ 的处理过程如下:

1) 算法 $B$ 随机地在 $\mathbb{Z}_{p}$ 中选取 $r_{1}, \cdots, r_{l}$, 且有 $r_{1}+\cdots+r_{l}=y$.

2) 对任意的 $i \in S_{\mathrm{ID}}$, 算法 $B$ 计算并返回 $r_{i} / t_{\mathrm{ID}_{i}, i}$ 给 $A$.

3) 对任意的 $i \in S_{s}$ 和 $i \neq l$, 攻击者 $A$ 秘密选取 $d_{i} \in \Sigma$ 作为其私有输入, 算法 $B$ 秘密计算 $\left\{r_{i} / t_{1, i}, \ldots, r_{i} / t_{n, i}\right\}$ 作为其私有输入, 然后共同运行 $O T_{n}^{1}$ 协议, 使得 $A$ 仅得到 $r_{i} / t_{d_{i}, i}$ 且算法 $B$ 无法 知道 $d_{i}$.

4) 当 $i=l$ 时, 与上一步唯一的不同是: 算法 $B$ 运行 $O T_{n}^{1}$ 协议的私有输入为 $\left\{r_{l} / t_{1, l}, \ldots, r_{l} / t_{n-1, l}\right.$, $\left.r_{l}\right\}$, 而其他过程完全相同.

$5)$ 上述过程结束后, 攻击者 $A$ 得到部分私钥 $d_{A}$, 且

$$
d_{A}=\left\{\left\{r_{i} / t_{\mathrm{ID}_{i}, i}\right\}_{i \in S_{\mathrm{ID}}},\left\{d_{i}, r_{i} / t_{d_{i}, i}\right\}_{i \in S_{s}-\{l\}},\left\{d_{l}, r_{l} / t_{d_{l}, l}: d_{l} \neq n\right\} \text { or }\left\{d_{l}, r_{l}: d_{l}=n\right\}\right\} .
$$

攻击者 $A$ 运行密钥有效性检测, 且该检测分为以下两种情况:

情况 1: 若 $d_{l} \neq n$, 则显然 $d_{A}$ 总是能通过检测.

情况 2: 若 $d_{l}=n$, 则 $d_{A}$ 仅在 $a=1$ 时可以通过猜测, 且由于 $a=1$ 的概率可忽略, 因此此时 $d_{A}$ 仅以可忽略的概率通过猜测, 即此时 $d_{A}$ 几乎总是无法通过检测.

6) 若 $d_{A}$ 未能通过密钥有效性检测, 则攻击者 $A$ 和算法 $B$ 均退出.

7) 算法 $B$ 运行 $I$ 的 Extract 算法, 生成身份信息 ID 的部分私钥 $d_{I}$ 给攻击者 $A$. 攻击者 $A$ 最后 得到 ID 的完整的私钥 $d_{\mathrm{ID}}=\left\{d_{I}, d_{A}\right\}$.

NewKeyGneration 阶段: 攻击者 $A$ 对其选取的挑战 ID, 以不可忽略的概率生成两个有效的私钥 $d_{\mathrm{ID}}^{1}$ 和 $d_{\mathrm{ID}}^{2}$, 且 $\operatorname{Trace}\left(d_{\mathrm{ID}}^{1}\right) \neq \operatorname{Trace}\left(d_{\mathrm{ID}}^{2}\right)$. 对任意的 $k \in\{1,2\}$, 令

$$
d_{\mathrm{ID}}^{k}=\left\{d_{I}^{k}, d_{A}^{k}=\left\{\left\{d_{A, i}^{k}\right\}_{i \in S_{\mathrm{ID}}},\left\{d_{i}^{k}, d_{A, i}^{k}\right\}_{i \in S_{s}}\right\}\right\} .
$$


则对给定的 $g^{a}$, 算法 $B$ 计算 $a$ 的过程如下:

1) 对任意的 $k \in\{1,2\}$, 算法 $B$ 运行密钥有效性检测过程, 检测 $d_{\mathrm{ID}}^{k}$ 的有效性; 若 $d_{\mathrm{ID}}^{k}$ 无效, 则算 法 $B$ 退出.

2) 对任意的 $k \in\{1,2\}$, 若 $d_{\mathrm{ID}}^{k}$ 均有 $d_{l}^{k} \neq n$, 则算法 $B$ 退出. 否则, 至少存在一个 $d_{\mathrm{ID}}^{k} \in\left\{d_{\mathrm{ID}}^{1}, d_{\mathrm{ID}}^{2}\right\}$ 有 $d_{l}^{k}=n$, 更进一步地, 若该 $d_{\mathrm{ID}}^{k}$ 中 $d_{A, l}^{k}=0$, 则算法 $B$ 也退出. 若算法 $B$ 没有退出, 则令 $d_{\mathrm{ID}}^{k} \in\left\{d_{\mathrm{ID}}^{1}, d_{\mathrm{ID}}^{2}\right\}$, 且满足 $d_{l}^{k}=n$ 和 $d_{A, l}^{k} \neq 0$. 算法 $B$ 如下计算 $a$ :

(1) 对任意的 $i \in\{1, \ldots, l-1\}$, 由于已知 $\left\{t_{\mathrm{ID}_{i}, i}\right\}_{i \in S_{\mathrm{ID}}} \cup\left\{t_{d_{i}^{k}, i}\right\}_{i \in S_{s}-\{l\}}$, 则算法 $B$ 为任意的 $i \in S_{\mathrm{ID}}$ 计算 $r_{i}^{k}=\left(d_{A, i}^{k}\right)^{t_{\mathrm{ID}_{i}, i}}$ 为任意的 $i \in S_{s}-\{l\}$ 计算 $r_{i}^{k}=\left(d_{A, i}^{k}\right)^{t_{d_{i}, i}^{k}, i}$.

(2) 然后由于已知 $y$, 算法 $B$ 计算 $r_{l}^{k}=y-\left(r_{1}^{k}+\cdots+r_{l-1}^{k}\right)$.

(3) 由于 $d_{\mathrm{ID}}^{k}$ 通过了密钥有效性检测且其 $d_{l}^{k}=n$, 则有 $\left(T_{n, l}\right)^{d_{A, l}^{k}}=g^{r_{l}^{k}}$ 成立. 进一步地, 由于公 开矩阵 $\boldsymbol{P} \boldsymbol{M}$ 中 $T_{n, l}=g^{a}$, 即 $g^{a \cdot d_{A, l}^{k}}=g^{r_{l}^{k}}$, 显然可以推出 $a \cdot d_{A, l}^{k}=r_{l}^{k}$. 因此算法 $B$ 可以通过计算 $r_{l}^{k} \cdot\left(d_{A, l}^{k}\right)^{-1}$, 有效求解 $a$.

以上描述即为算法 $B$ 的整个过程. 显然算法 $B$ 的正确性是很容易验证的, 并且可以在有效时间 内停止. 下面将分别简要分析: 在算法 $B$ 不退出的条件下, 其构造的环境和真实的新的通用 A-IBE 方 案不可区分; 以及算法 $B$ 成功求解 DL 问题的概率.

在算法 $B$ 的初始化阶段, 所有的公开参数和真实的新的通用 A-IBE 方案的 Setup $-u A$ 算法的输 出具有相同的分布; 其 KeyGeneration 阶段, 若算法 $B$ 没有退出 (即攻击者 $A$ 成功的通过了私钥有效 性检测, 且其运行 $O T_{n}^{1}$ 协议的私有输入 $d_{l}$ 不等于 $\left.n\right)$, 则显然有算法 $B$ 发送给攻击者 $A$ 的响应数据 均和真实环境下的 KeyGeneration 协议具有相同分布. 因此, 只要算法 $B$ 没有退出, 则其构造的环境 和真实的新的通用 A-IBE 方案的环境不可区分.

算法 $B$ 成功求解 DL 问题的概率主要由算法 $B$ 不退出的概率确定. 下面将分别计算 KeyGeneration 阶段和 NewKeyGeneration 阶段中, 算法 $B$ 退出的概率:

KeyGeneration 阶段: 根据算法 $B$ 的构造可以看出, 每一次的私钥生成过程和其他已生成的私钥 是相互独立的, 甚至同一个用户的不同私钥也是相互独立的. 因此每一次私钥的生成过程中, 由攻击 者 $A$ 选择的 $d_{l} \neq n$ 的概率为 $(1-1 / n)$; 那么对于 $q_{\mathrm{ID}}$ 次私钥的生成过程而言, 算法 $B$ 不退出的概率 为 $(1-1 / n)^{q_{\mathrm{ID}}}$. 显然该概率小于 Goyal 提出的 A-IBE-G2 方案的安全性证明中相同阶段的算法 $B$ 不 退出的概率 (A-IBE-G2 方案在此处的概率为 $\left.(1-1 / 2)^{q_{\mathrm{ID}}[10]}\right)$.

NewKeyGeneration 阶段: 在安全性定义 ComputeNewKey game ${ }^{[10]}$ 下, 令攻击者 $A$ 以不可忽略的 概率 $\varepsilon$ 有效的计算出挑战 ID 的两个有效的私钥 $d_{\mathrm{ID}}^{1}$ 和 $d_{\mathrm{ID}}^{2}$, 且有 $\operatorname{Trace}\left(d_{\mathrm{ID}}^{1}\right) \neq \operatorname{Trace}\left(d_{\mathrm{ID}}^{2}\right)$. 假设该阶 段算法 $B$ 不退出, 则该事件的成立等价于下面 3 个事件同时成立, 分别如下:

事件 1: 攻击者 $A$ 生成的私钥 $d_{\mathrm{ID}}^{1}$ 和 $d_{\mathrm{ID}}^{2}$ 通过密钥有效性检测, 且该事件成功的概率为 $\varepsilon$.

事件 2: 至少存在一个私钥 $d_{\mathrm{ID}}^{k} \in\left\{d_{\mathrm{ID}}^{1}, d_{\mathrm{ID}}^{2}\right\}$ 满足 $d_{l}^{k}=n$. 由于所有的私钥生成是相互独立的, 且 公开矩阵 $\boldsymbol{P} \boldsymbol{M}$ 中所有元素具有相同分布, 则该事件的成功概率 $1 / n$.

事件 3: 在事件 2 成立的基础上, $d_{\mathrm{ID}}^{k}$ 的 $d_{A, l}^{k}$ 不等于 0 . 由于 $\left\{d_{A, i}^{k}\right\}_{i \in\{1, \ldots, l\}}$ 中至少有一个 $d_{A, i}^{k}$ 不 等于 0 , 且公开矩阵 $\boldsymbol{P} \boldsymbol{M}$ 中所有元素具有相同分布, 则该事件的成功概率为 $1 / l$.

综上所述, 算法 $B$ 不退出的概率为 $(1-1 / n)^{q_{\mathrm{ID}}} \frac{\varepsilon}{n \cdot l}$. 显然有, 当 $n=q_{\mathrm{ID}}$ 时, 该概率最低, 即 $(1-1 / n)^{q_{\mathrm{ID}}} \frac{\varepsilon}{n \cdot l} \approx \varepsilon /\left(e \cdot q_{\mathrm{ID}} \cdot l\right)(e \approx 2.71)$. 因此算法 $B$ 不退出的概率不可忽略, 也就是说, 算法 $B$ 可以 以不可忽略的概率求解 DL 问题.

显然上述结论和 DL 假设成立相矛盾, 因此在安全性定义 ComputeNewKey game ${ }^{[10]}$ 下, 不存在 


\section{表 1 A-IBE-G2 方案和新的通用 A-IBE 方案的安全性比较}

\begin{tabular}{cccc}
\hline & 难题 & 归约程度 & 安全性定义 \\
\hline A-IBE-G2 方案 & 计算的 $\mathrm{MDH}$ 假设 ${ }^{[10]}$ & $\frac{1}{2}\left(1-\frac{1}{2}\right)^{q_{\mathrm{ID}} \varepsilon}$ & The Selective-ID ComputeNewKey game \\
新的通用 A-IBE 方案 & DL 假设 & $\varepsilon /\left(e \cdot q_{\mathrm{ID}} \cdot l\right)(e \approx 2.71)$ & The ComputeNewKey game \\
& & (最优值) & (adaptively selected ID) \\
\hline
\end{tabular}

攻击者能够有效地和不可忽略地概率成功攻破新的通用 A-IBE 方案. 得证.

\section{5 分析}

对了说明和突出体现新的通用 A-IBE 方案的优点, 本节将对该提出方案进行若干重要方面的分 析和对比. 与此同时, 为了公正性, 本文还将指出该新方案的不足.

\section{1 安全性的归约程度}

在现代密码学中, 一个可证明安全的方案意味着: 在一定的安全性定义下, 一个难题的求解可以 有效的被归约到攻击者对该方案的成功的攻击, 因此若此时攻击者可以有效地攻破该方案, 则该难题 也能有效地被求解. 相反地, 由于难题通常认为是不可解的, 因此在该安全性定义下, 并不存在攻击者 可以有效地攻破该方案, 即该方案是可证明安全的. 更进一步地抽象地说, 通过这一种归约, 从 3 个方 面, 即难题的类型、归约程度和安全性定义, 实现了对该方案的安全性量化. 换一个角度说, 这 3 个方 面也共同决定了密码方案的安全性退化程度. 因此在应用中, 为了弥补这种安全性退化需要选择较大 的安全参数, 而且退化程度越高, 相应地安全参数选取地越大. 因此如何减少安全性的退化成为现代 密码学的重要研究领域.

从上述 3 个决定安全性退化的方面, 表 1 对比了本文提出的新的通用 A-IBE 方案和 Goyal 提出 的 A-IBE-G2 方案 [10]. 由于新的通用 A-IBE 方案基于的难题比 A-IBE-G2 方案更难, 同时, 由于新的 通用 A-IBE 方案拥有更 “紧” 的归约程度、基于更强的安全性定义, 因此本文的提出方案, 即新的通 用 A-IBE 方案, 具有更少的安全性退化.

由于新的通用 A-IBE 方案具有更少的安全性退化, 因此它可以选择更小的安全参数, 从而提高计 算效率. 换句话说, 当上述两个方案选取相同大小的安全参数时, 新的通用 A-IBE 方案比 A-IBE-G2 方案具有更高的安全性.

\section{2 对运算的次数}

通过对 MOV 归约理论 ${ }^{[11]}$ 的形式化总结, Boneh 首次提出了对运算的概念, 并且基于该对运算 成功地构造了第一个实用的和可证明安全的 IBE 方案. 当前对运算在密码学领域有着广泛地应用, 特

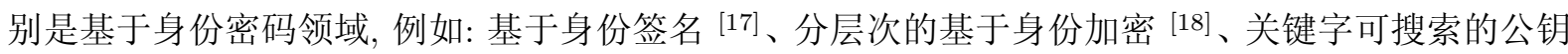
加密 [19] 等等. 但是由于对运算比常规的群中模幂运算的时间开销大得多, 因此如何减少对运算的次 数成为提高密码方案效率的重要方法之一.

根据第 3 节对新方案的表述可以看出, 新的通用 A-IBE 方案可以将对运算的次数减少为 2 次; 而 且通过表 2 的对比可以看出, 由于 A-IBE-G2 方案的对运算次数为 $2 l+2$ 次, 其中 $l$ 为关于安全参数 $k$ 的多项式, 因此在对运算次数方面, 新的通用 A-IBE 方案要高效地多. 
表 2 A-IBE-G2 方案和新的通用 A-IBE 方案的对运算次数比较

\begin{tabular}{cccc}
\hline & $\begin{array}{c}\text { 密钥生成协议的密钥 } \\
\text { 有效性检测阶段 }\end{array}$ & $\begin{array}{c}\text { 解密算法的密文有效 } \\
\text { 性检测阶段 }\end{array}$ & $\begin{array}{c}\text { 解密算法的明文 } \\
\text { 恢复阶段 }\end{array}$ \\
\hline A-IBE-G2 方案 & $l$ 次 & 2 次 (最优值) & $l$ 次 \\
新的通用 A-IBE 方案 & 0 次 & 2 次 (最优值) & 0 次 \\
\hline
\end{tabular}

表 3 在密钥生成阶段 A-IBE-G2 方案和新的通用 A-IBE 方案的时间复杂度比较

\begin{tabular}{|c|c|c|c|c|c|c|c|c|}
\hline & \multicolumn{8}{|c|}{ 密钥生成阶段 } \\
\hline & \multicolumn{5}{|c|}{ 密钥的计算 } & \multicolumn{3}{|c|}{ 密钥有效性检测 } \\
\hline & \multicolumn{2}{|c|}{$\mathbb{Z}_{p}$} & \multirow{2}{*}{$O T_{2}^{1}$} & \multirow{2}{*}{$O T_{n}^{1}$} & \multirow{2}{*}{$\begin{array}{l}G_{1} \\
x^{y}\end{array}$} & \multicolumn{2}{|c|}{$G_{1}$} & \multirow{2}{*}{$\frac{G_{1}}{e(x, y)}$} \\
\hline & $x \cdot y$ & $x^{-1}$ & & & & $x \cdot y$ & $x^{y}$ & \\
\hline A-IBE-G2 方案 & $l_{\mathrm{ID}}+2 l_{s}$ & $l_{\mathrm{ID}}+2 l_{s}$ & 1 & 0 & $l_{\mathrm{ID}}+2 l_{s}$ & $l-1$ & 0 & $l$ \\
\hline 新的通用 A-IBE 方案 & $l_{\mathrm{ID}}+n \cdot l_{s}$ & $l_{\mathrm{ID}}+n \cdot l_{s}$ & 0 & 1 & 0 & $l-1$ & $l$ & 0 \\
\hline
\end{tabular}

在新的通用 A-IBE 方案中, 仅仅只有两次对运算, 而且可以容易的看出该次数不可能再减少. 抽 象地说, 密文有效性检测是一个求解 DDH 问题的过程, 而当前仅有两种方法可以做到: 一种方法是最 原始的方法, 即接收者已知 DDH 问题的一个秘密时, 他就可以求解 DDH 问题 (此处的秘密可以看成 是接收者的私钥或其一部分); 另一种方法是即使接收者不知道有关 DDH 问题秘密, 他可以通过对运 算求解 DDH 问题. 根据新的通用 A-IBE 方案中 Encrypt $-u A$ 算法的构造可以看出, 接收者不可能知 道所有的秘密, 从而帮助他完成密文有效性检测, 因此需要由对运算来有效实现该检测过程. 当然理 论上, 对运算的功能可以被零知识证明代替, 但是没有人会怀疑后者通常是非常复杂的.

更进一步地, 为什么密文有效性检测阶段需要至少两次对运算呢? 在解释以前首先需要明确重要 的一点: 密文有效性检测是一个确定算法, 它的输出为 1 bit 信息. 但是由于密文有效性检测的输入必 然是具有随机性的信息 (因为这些信息来至密文), 而对运算并不会剔除输入的随机性, 因此抽象地讲, 它需要至少两次对运算然后组合处理这些对运算的结果来剔除随机性, 从而得到 1 bit 的确定信息来 完成检测.

\section{3 时间复杂度分析}

为了直观的说明新的通用 A-IBE 方案在时间复杂度方面的优势, 本节通过对比 A-IBE-G2 方案, 列举出各种重要的密码学操作的次数. 在进行比较之前, 为了简化分析过程, 本文首先假设上述两个 方案具有相同长度的安全参数. 甚至, 我们可以认为这两个方案中具有相同功能的群是完全一样地, 而这一点在上述的假设下, 并不会影响我们对时间复杂度的分析.

在前述的假设下, 通过分析新的通用 A-IBE 方案和 A-IBE-G2 方案, 表 3 和表 4 分别列出了 3 个 阶段中上述两个方案的几种重要的密码操作的次数 (其中没有列出有关 IBE 方案的密码操作次数, 因 为 IBE 方案作为上述两个方案的组成部分可以是完全相同的; 也没有列出解密阶段密文有效性检测 的密码操作次数, 因为该检测在上述两个方案中也是完全相同的). 为了清晰的表达, 额外定义如下符 号: 分别令 $x \cdot y, x^{-1}$ 和 $x^{y}$ 表示某个群的乘法、求逆和模幂运算; 令 $e(x, y)$ 表示对运算; 令函数 $T(\alpha, \beta)$ 的输入分别为群的运算和群, 输出为该运算的时间复杂度, 例如: $T\left(x \cdot y, \mathbb{Z}_{p}\right)$ 的输出为群 $\mathbb{Z}_{p}$ 的乘法运 算的时间复杂度.

从表 3 可以看出, 当 $n=2$ 时, 在密钥生成阶段新的通用 A-IBE 方案明显优于 A-IBE-G2 方案. 
表 4 在加密阶段和解密阶段 A-IBE-G2 方案和新的通用 A-IBE 方案的时间复杂度比较

\begin{tabular}{ccccccccc}
\hline & \multicolumn{3}{c}{ 加密阶段 } & \multicolumn{3}{c}{ 解密阶段 (不考虑密文有效性检测) } \\
\cline { 2 - 8 } & \multicolumn{3}{c}{$G_{1}$} & $G_{1}$ & & \multicolumn{1}{c}{$G_{1}$} & \\
\hline & $x \cdot y$ & $x^{y}$ & $x \cdot y$ & $x^{-1}$ & $x^{y}$ & $x \cdot y$ & $x^{-1}$ & $e(x, y)$ \\
A-IBE-G2 方案 & 1 & $2+l_{\mathrm{ID}}+2 l_{s}$ & 0 & 0 & 0 & $l$ & 1 & $l$ \\
新的通用 A-IBE 方案 & 1 & $2+l_{\mathrm{ID}}+n \cdot l_{s}$ & $l$ & 1 & $l$ & 0 & 0 & 0 \\
\hline
\end{tabular}

表 5 A-IBE-G2 方案和新的通用 A-IBE 方案在存储量和通讯量的比较

\begin{tabular}{ccc}
\hline & 初始化阶段的公开参数 & 密钥生成协议的不经意传输 \\
\hline A-IBE-G2 方案 & $O(2 l)$ & $O T_{2}^{1}$ \\
新的通用 A-IBE 方案 & $O(n \cdot l)$ & $O T_{n}^{1}$ \\
\hline
\end{tabular}

更具体地说, $n=2$ 时, 新的通用 A-IBE 方案在密钥生成阶段的时间复杂度比 A-IBE-G2 方案少了 $l_{s} \cdot T\left(x^{y}, G_{1}\right)+l \cdot T\left(e(x, y), G_{1}\right)$. 并且随着 $n$ 的增加, 只要 $O T_{n}^{1}$ 协议和 $O T_{2}^{1}$ 协议的时间复杂度差异小 于 $l_{s} \cdot T\left(x^{y}, G_{1}\right)+l \cdot T\left(e(x, y), G_{1}\right)$, 那么新的通用 A-IBE 方案将总是优于 A-IBE-G2 方案. 关于 $O T_{n}^{1}$ 协议和 $O T_{2}^{1}$ 协议时间复杂度差异的细节可以参考 ${ }^{[15]}$ (注意: 此处的时间复杂度计算, 合理地忽略了 群的乘法和求逆运算).

相似地, 从表 4 可以看出, 当 $n=2$ 时, 在加密和解密的时间复杂度方面, 新的通用 A-IBE 方案 比 A-IBE-G2 方案少了 $l \cdot\left[T\left(e(x, y), G_{1}\right)-T\left(x^{y}, G_{1}\right)\right]$. 并且随着 $n$ 的增加, 只要 $(n-2) \cdot l_{s} \cdot T\left(x^{y}, G_{1}\right)$ 小于 $l \cdot\left[T\left(e(x, y), G_{1}\right)-T\left(x^{y}, G_{1}\right)\right]$, 则新的通用 A-IBE 方案将总是优于 A-IBE-G2 方案 (在此合理的 忽略了群的乘法和求逆运算的时间复杂度).

因此, 从上述分析可以直观地看出: 在假设新的通用 A-IBE 方案和 A-IBE-G2 方案具有相同长度 的安全参数的条件下, 当 $n=2$ 时, 新的通用 A-IBE 方案在时间复杂度方面最优于 A-IBE-G2 方案, 但是随着 $n$ 的增加该优势会逐渐减少.

但是实际上, 通过 5.1 小节的分析可以看出, 在新的通用 A-IBE 方案和 A-IBE-G2 方案具有相同 的安全强度时, 新的通用 A-IBE 方案可以选取小得多的安全参数, 因此在去掉上述中给出的特殊假设 时, 新的通用 A-IBE 方案将具有比上述更优秀的性能, 其随着 $n$ 的增加所造成的性能优势的减少将 更慢.

综上所述, 新的通用 A-IBE 方案在选取适当的参数时可以比 A-IBE-G2 方案高效得多.

\section{4 缺陷分析}

虽然新的通用 A-IBE 方案具有很多明显的优势, 但是其在存储量和通讯量方面的缺陷也很明显. 通过对比新的通用 A-IBE 方案和 A-IBE-G2 方案在存储量和通讯量, 可以发现这两个方案主要的不 同集中在公开参数和不经意传输.

从表 5 可以看出, 新的通用 A-IBE 方案的公开参数的空间复杂度是 A-IBE-G2 方案的 $n / 2$ 倍. 上 述两个方案的通讯量差异集中在各自使用的不经意传输的不同, 由于该内容超出本文的范围, 因此具 体细节可以参考文献 ${ }^{[15,16]}$.

因此根据表 5 , 当选择一个较小的 $n$ 时, 可以有效的减少新的通用 A-IBE 方案的存储量和通讯量, 但是相应地, 一个较小的 $n$ 将增加新方案的安全性退化, 所以实用中需要选择一个折衷的或满足特定 需求的大小的 $n$, 进而平衡利弊. 


\section{6 总结}

鉴于 IBE 方案存在密钥托管问题, 2007 年 Goyal 创造性地提出一种新的方法用于降低 IBE 方案 中用户对 PKG 的信任程度, 且该方案称为第三方权利受约束的 IBE 方案, 简称为 A-IBE 方案. 本文 在认真研究 Goyal 提出的一个通用 A-IBE 方案 (简称为 A-IBE-G2) 的基础上, 提出了一个新的通用 A-IBE 方案, 该新方案不仅在很大程度上减少了对运算次数, 而且降低了安全性退化. 具体地说, 在对 运算次数方面, 新的通用 A-IBE 方案将对运算次数减少到 2 次, 且通过简要地证明, 证明了该次数达 到最优; 在安全性退化方面, 与 A-IBE-G2 方案相比, 新的通用 A-IBE 方案基于更弱的难题 (DL 问题), 在更强的安全性定义下 (攻击者可以适应性选择挑战身份) 具有更 “紧” 的安全性归约, 因此新的通用 A-IBE 方案可以选取更小的安全参数, 从而在一定程度上提高该方案的执行效率. 在时间复杂度方面, 通过对比分析新的通用 A-IBE 方案和 A-IBE-G2 方案, 可以看出新方案在选取适当大小的参数后, 其 优势将明显高于 A-IBE-G2 方案, 而且由于新方案可以选取更小的安全参数, 因此这一优势将进一步 地提高.

但是与此同时, 新的通用 A-IBE 方案的这些优势也在一定程度上增加了公开参数的存储量, 和执 行 $O T_{n}^{1}$ 协议时的通讯量. 因此在实际应用中, 可以选取折哀或是满足特定需求的参数大小, 进而平衡 利弊.

\section{参考文献}

1 Shamir A. Identity-based cryptosystems and signature schemes. In: Advances in Cryptology- Proceedings of CRYPTO'84. LNCS 196. California: Springer-Verlag, 1985. 48-53

2 Boneh D, Franklin M. Identity-based encryption from the weil pairing. In: Advances in Cryptology-Crypto 2001. LNCS, Vol 2139. California: Springer-Verlag, 2001. 231-229

3 Boneh D, Boyen X. Efficient selective-ID identity based encryption without random oracles. In: Advances in CryptologyEUROCRYPT'2004. LNCS, Vol 3027. Switzerland: Springer-Verlag, 2004. 223-238

4 Boneh D, Boyen X. Secure identity based encryption without random oracles. In: Advances in Cryptology-Crypto 2004. LNCS, Vol 3152. California: Springer-Verlag, 2004. 443-459

5 Waters B. Efficient identity-based encryption without random oracles. In: Advances in Cryptology-EUROCRYPT'2005. LNCS, Vol 3494. Denmark: Springer-Verlag, 2005. 114-127

6 Gentry C. Practical ientity-based encyrption without random oracles. In: Advances in Cryptology-EUROCRYPT'2006. LNCS, Vol 4004. Russia: Springer-Verlag, 2006. 445-464

7 Baek J, Zheng Y L. Identity-based threshold decryption. In: Public-Key Cryptography'2004. LNCS, Vol 2947. Singapore: Springer-Verlag, 2004. 262-276

8 Sahai A, Waters B. Fuzzy identity-based encryption. In: Advances in Cryptology- EUROCRYPT'2005. LNCS, Vol 3494. Denmark: Springer-Verlag, 2005. 457-473

9 Al-Riyami S, Paterson K. Certificateless public key cryptography. In: Advances in Cryptology -Asiacrypt'2003. LNCS, Vol 2332. Taibei: Springer-Verlag, 2003. 452-473

10 Goyal V. Reducing trust in the PKG in identity-based Cryptosystems. In: Advances in Cryptology-Crypto 2007. LNCS, Vol 4622. California: Springer-Verlag, 2007. 430-447

11 Menezes A J, Okamoto T, Vanstone S A. Reducing elliptic curve logarithms to logarithms in a finite field. IEEE Trans Inform Theory, 1993, 39: 1639-1646

12 Bao F, Robert H D, Zhu H F. Variations of diffie-hellman problem. In: ICICS 3. LNCS, Vol 2836. Singapore: Springer-Verlag, 2003. 301-312

13 Mao W B. Modern Cryptography: Theory and Practice. Upper Saddle River: Prentice Hall, 2003. 252-254

14 Lu C J. On the security loss in cryptographic reductions. In: Advances in Cryptology-EUROCRYPT'2009. LNCS, Vol 5479. Germany: Springer-Verlag, 2009. 72-87 
15 Tzeng W G. Efficient 1-out-of-n oblivious transfer schemes with universally usable parameters. IEEE Trans Comput, 2004, 53(2): 232-240

16 Naor M, Pinkas B. Oblivious transfer and polynomial evaluation. In: Proceedings of 31st ACM Symposium on Theory of Computing. Atlanta: Elsvier, 1999. 145-254

17 Boneh D, Boyen X. Short signatures without random oracles. In: Advances in Cryptology- EUROCRYPT'2004. LNCS, Vol 3027. Switzerland: Springer-Verlag, 2004. 56-73

18 Gentry C, Silverberg A. Hierarchical ID-based cryptography. In: Advances in Cryptology- Asiacrypt'2002. LNCS, Vol. 2501. New Zealand: Springer-Verlag, 2002, 548-566

19 Abdalla M, Bellare M, Catalano D, et al. Searchable encryption revisited: consistency properties, relation to anonymous IBE, and extensions. In: Advances in Cryptology- CRYPTO'2005. LNCS, Vol 3621. California: Springer-Verlag, 2005. $205-222$ 